- FOR̊TBILD'UNG . KRITISCH GELESEN

\title{
Riechen Sie den Spargel im Urin?
}

\section{Hier steht eine Anzeige.}

\section{Nach dem Spargelgenuss riecht der Urin eigenartig. Manche Leute bemerken das aber nicht. Nun wissen wir endlich den Grund.}

Viele Menschen sind nicht in der Lage, den schwefeligen Geruch der Spargel-Metaboliten Methanthiol und S-Methyl-Thioester im Urin zu riechen. Erst vor wenigen Jahren wurde bewiesen, dass es sich dabei um eine spezifische Anosmie handelt.

Nun wollten ein internationales Team von Epidemiologen und Humangenetiker den genetischen Hintergrund dieses Phänomens klären. Dafür wurden Daten von 6.909 kaukasischen Personen aus der Nurses' Health Study und der Health Professionals Follow-up Study neu ausgewertet. Für alle lagen genetische Daten aus genomweiten Assoziationsstudien vor. Sie wurden nun zu ihrer Fähigkeit befragt, den Spargelgeruch wahrzunehmen. 58\% der Männer und $61,5 \%$ der Frauen hatten eine entsprechende Anosmie.

Die Genanalyse ergab drei unabhängige Marker in derselben Region des Chromosoms 1, die mit der SpargelAnosmie assoziiert waren: rs13373863, rs71538191 und rs6689553.

- Markt SC, Nuttall E, Turman C et al. Sniffing out significant "Pee values": genome wide association study of asparagus anosmia. BMJ. 2016:355:6071

\section{KOMMENTAR}

So, diese Frage wäre nun auch geklärt obwohl es noch interessant wäre, wie es bei anderen Ethnien damit bestellt ist. Vorerst gilt: Lassen Sie sich nicht aus der Ruhe bringen, wenn Sie nach dem Genuss von Spargel keine Veränderung ihres Uringeruchs feststellen. Jedem Zweiten geht es ebenso.

\section{Springer}

